\title{
MedienPädagogik
}

Zeitschrift für Theorie und Praxis der Medienbildung

\section{Medienethik: Werte neu denken}

\section{- Gibt es Massstäbe im Informationszeitalter?}

Claudia de Witt

Im Übergang von der Informations- zur Wissensgesellschaft erweist sich die Frage nach pädagogischen Massstäben als besonders relevant. Zwar entstehen mit der Entwicklung zur Globalisierung durch die neuen Medien neue Handlungsmöglichkeiten, zugleich aber auch Pluralität und Unübersichtlichkeit bei den Wertorientierungen. Eine Auseinandersetzung mit medienethischen Fragen muss deshalb auf Überlegungen zielen, Werte neu zu denken. Die folgenden Beiträge sind auf dem Hintergrund der Frühjahrstagung der Kommission Medienpädagogik 2001 in Mainz entstanden. In Form von Thesen haben sich die Autoren aus unterschiedlichen Perspektiven mit den ethischen Herausforderungen durch die alten und neuen Medien auseinandergesetzt. Auf diesem Hintergrund ist ein facettenreicher Beitrag zur Medienethik entstanden.

- Rüdiger Funiok, München

- Dieter Spanhel, Nürnberg

- Ben Bachmair, Kassel

- Theo Hug, Innsbruck

- Bardo Herzig und Gerhard Tulodziecki, Paderborn

- Manfred Lang, Kiel

- Rudolf Kammerl, Passau 


\section{MedienPädagogik}

Zeitschrift für Theorie und Praxis der Medienbildung

\section{Medienethik - wie kommt sie zu konsensfähigen Massstäben?}

Rüdiger Funiok

\section{Mensch - Medien - Moral: Gibt es noch einen verbindlichen Rahmen?}

Während die Medien immer mehr zum aktuellen Menschsein gehören, scheint es mit der Moral umgekehrt zu sein. Sie ist in mancher Hinsicht auf dem Rückzug. Wir sind uns nicht mehr sicher, wo welche Normen gelten, wo moralische Überzeugungen überhaupt noch relevant sind. Wir fragen uns heute: Wie lassen sich der demokratische Minimalkonsens und die breit akzeptierte Alltagsmoral auf den Bereich der Medienkommunikation anwenden? Nach welchen Kriterien lässt sich bestimmen, ob Medienangebote noch angängig oder moralisch bedenklich sind (z.B. im Blick auf Pornographie)?

\section{Medienethik ist eine (umfassende) Bereichsethik, welche prinzipienorientierte} Argumentation mit empirischen Aussagen verbindet

Man sagt, Medienethik sei eine «Bereichsethik» - wie die Umweltethik, die Medizinethik und die Wirtschaftsethik. Aber in gewisser Weise sind Medien ein ubiquitärer Bereich, eine Bedingung unserer Weltwahrnehmung: Was wir von der Welt wissen, wissen wir durch die Medien. Was hier erlaubt ist und was nicht, ergibt sich für eine reflektierte Medienethik nicht aus den Geschmacksurteilen einer tonangebenden Gruppe (z.B. des Bildungsbürgertums), sondern ist im Blick auf die Grundrechte Betroffener, z.B. von Minderheiten zu bestimmen. Dass das nutzende Publikum seine Selbstbestimmung und Autonomie gegen die «Faszination und Vereinnahmungstendenzen der Medien» (Jäckel 1999) wahren und weiterentwickeln sollte - diese Forderung ist ebenso alt wie es Erziehung im neuzeitlichen Sinne und das entsprechende Bemühen um Selbstbildung gibt. Das alles sind keine grundsätzlich neuen moralischen Herausforderungen. Sie stellen sich heute nur mit besonderer Dringlichkeit, weil unsere Welt immer stärker von Medien durchdrungen und geprägt wird. Deshalb ist Medienethik, die Frage nach der Verantwortung im Medienhandeln, als eigene Bereichsethik notwendig.

Worin die konkreten Gefahren für die Demokratie und die Entwicklung des einzelnen bestehen, lässt sich übrigens nicht nur durch den Bezug auf eine konsensfähige Moral festlegen. Es ist Aufgabe einer entsprechend differenzierten Medienforschung aufzuzeigen, wo bei einzelnen Bevölkerungsgruppen (z.B. Kindern) Irritationen und Orientierungsprobleme liegen. Neben dem Bezug zur Wertebene hat Medienethik also auch empirische Aussagen aufzugreifen. 


\section{Medienethik vermag anthropologisch zu argumentieren}

Die alte ontologische Anthropologie ist - wegen des naturalistischen Fehlschlusses - passé. Aber die anthropologische Reflexion kann heute sprachanalytisch verfahren, also auf die Grammatik unseres Denkens und Redens über die Grundlagen eines verantwortlichen Zusammenlebens zurückgreifen. Dabei lassen sich nach Rehbock $(1997,73)$ durchaus Aussagen über eine grundlegende Moral formulieren, die universal und - ihrem Anspruch nach, nicht was ihre geschichtliche Ausgestaltung und faktische Befolgung betrifft - invariabel sind. Solchermassen fundamentalmoralische Aussagen seien nicht - «szientistisch» - durch ein Aufgreifen empirischer Ergebnisse (der Medizin, der Ethnologie, der Psychologie oder Soziologie) zu gewinnen, auch nicht - «ontologistisch» - durch die dogmatische Fixierung einer bestimmten (z.B. christlichen) Auffassung vom Menschen und seinen Werten, sondern indem elementare Kategorien unserer Moralsprache und Selbstverständlichkeiten unserer moralischen Praxis bewusst gemacht werden. Gemeint sind «Lebens- und Praxisformen, die ... wir gar nicht sinnvoll bezweifeln können, wenn wir uns selbst, unsere Existenz und unser Selbstverständnis als Menschen, nicht vollkommen in Zweifel ziehen wollen.» (S. 81)

Dazu gehören: Personalität «als ein wesentlicher Aspekt unserer Einstellung zum Anderen als Mensch» (S. 75), indem wir nicht nur negativ «die Autonomie des Anderen als Person .. achten», sondern ihm auch positiv «Zuwendung» und "Fürsorge» zukommen lassen (S. 99). Dazu gehören: Leiblichkeit, Sterblichkeit, das Angewiesensein auf Kommunikation, soziale Bedürftigkeit, die Bereitschaft zum Einsatz für Gemeinwohl und Gerechtigkeit.

\section{Ein gültiger Ausgangspunkt: Achtung und Respekt vor der Würde jedes Menschen} Wer diesen moralischen Unterstellungen oder Erwartungen, wie sie in unserer Gesellschaft an das Verhalten von Menschen herangetragen werden, zuwider handelt, der erfährt selbst «Nichtachtung» oder im positiven Fall «Achtung». Diese positive oder negative Bewertung macht nach Niklas Luhmann (1984, 318 f.; 1990, 17 f.) den «Code» der moralischen Kommunikation aus.

Die von Luhmann nur en passant aufgezählten moralischen «Unterstellungen» sind deutlicher ausgeführt in den (auch international zunehmend verbindlichen) Menschenrechtserklärungen (vgl. Gert 1966; Ropohl 1999). Die Menschenrechte bilden auch das Fundament der demokratischen Medienordnung: Meinungsäusserung- und Pressefreiheit; sie sind zwar nur als formale Freiheiten institutionalisiert und bedürfen der inhaltlichen Füllung durch mündige Staatsbürger (vgl. Deussen 1994, 156). «Dennoch sollte der Wert, den solche Grundeinstellungen für die Medienethik haben, nicht unterschätzt werden. Sie dienen im medienethischen Diskurs als ein normativer Rahmen, der nicht ohne weiteres in Frage gestellt werden darf.» (Kaminsky 2000, 50) 


\section{Anwendungsfall Pornographie (im Fernsehen und Internet)}

Wenden wir das auf das Problem der Pornographie und den Jugendschutz an! In individualethischer Perspektive gilt heute als guter ein Gebrauch von Pornographie durch den Einzelnen dann als problematisch, wenn die Darstellung und die individuelle Verarbeitung ein sexuelles Verhalten nahelegt, in dem sich eine Verachtung der Menschenwürde der dargestellten Personen manifestiert.

In sozialethischer Perspektive geht es einmal um den Schutz vor unerwünschter Konfrontation, also den Schutz der Privatsphäre: gegen die erzwungene Wahrnehmung sexueller Darstellungen oder Praktiken an öffentlich zugänglichen Orten, ohne explizit geäusserten Wunsch des Adressaten. Der zweite Gesichtspunkt ist der Schutz der sexuellen und geistig-moralischen Entwicklung Heranwachsender, der Jugendschutz: So «ist eine pornographische Darbietung vor Kindern und Jugendlichen dann ethisch nicht zu rechtfertigen, wenn sie eine Einstellung zu sexuellem Verhalten suggeriert, die mit dem Respekt vor der Würde des Individuums nicht vereinbar ist.» (Feldhaus/Molinski 1998, 40)

Dabei stehen pornographische Darstellungen im Fernsehen - nach einer Interpretation des Supreme Court der USA - stärker im öffentlich, allgemein zugänglichen Raum als das beim Internet der Fall ist, welches zur Kategorie der Zeitungen und Bücher zählt, die man im Normalfall explizit erwirbt oder beim Herumliegen willentlich öffnet. Staatliche Institutionen sollten dahingehend zusammenwirken, dass eine angemessene sexuelle Sozialisation in Familie und Öffentlichkeit möglich bleibt und die Jugendlichen wie ihre Erziehungspersonen dabei unterstützt werden.

\section{Literatur}

Deussen, G. (1994): «Ethisches Minimum». Grundwerte öffentlicher Kommunikation in einer pluralistischen Gesellschaft. In: Wolfgang Wunden (Hrsg.), Öffentlichkeit und Kommunikationskultur. (Beiträge zur Medienethik, Band 2). Frankfurt a.M., S. 151-163. Gemeinschaftswerk der Evangelischen Publizistik.

Feldhaus, St./ Molinski, W. (1998): Art. Pornographie, 3. Ethisches Lexikon der Bioethik. (Hrsg. im A. der Görresgesellschaft von W. Korff, L. Beck u. P. Mikat) Bd. 3. Gütersloh, S. 38-41.

Gert, B. (1966): Die moralischen Regeln. Frankfurt a.M.

Kaminsky, C. (2000): Medienethik - Ein Engagement zwischen Verunsicherung und Verantwortung. In: Schicha, C./ Brosda, C. (Hrsg.): Medienethik zwischen Theorie und Praxis. Nomen für die Kommunikationsgesellschaft. Münster, S. 43-52.

Luhmann, N. (1984): Soziale Systeme. Grundriss einer allgemeinen Theorie. Frankfurt a.M. Luhmann, N. (1990): Paradigm lost: Über die ethische Reflexion der Moral. Rede anlässlich der Verleihung des Hegel-Preises 1989. Frankfurt a.M.

Rehbock, Th. (1997): Warum und wozu Anthropologie in der Ethik? In: Wils, J.-P. (Hrsg.): Anthropologie und Ethik. Biologische, sozialwissenschaftliche und philosophische Überlegungen. Tübingen \& Basel, S. 64-109.

Ropohl, G. (1999): Welche Schwierigkeiten die Technik mit der Ethik hat. In: Kampits, P./ Weiberg, A. (Hrsg.): Angewandte Ethik/ Applied Ethics. Wien, S. 235-246. 


\section{MedienPädagogik}

Zeitschrift für Theorie und Praxis der Medienbildung

\section{Thesen zu ethischen Grundfragen der Medienpädagogik}

Dieter Spanhel

\section{Ausgangslage}

Neue Medien und die damit einhergehende zunehmende Flut an Medienangeboten, erleichterter Zugang, erweiterte Verfügungsmöglichkeiten und immer grössere Reichweiten der Medien (Globalisierung) eröffnen neue Handlungsmöglichkeiten, erhöhen aber auch den ständigen Zwang, sich entscheiden und auswählen zu müssen.

Die modernen Medien stellen Öffentlichkeit als einen Raum der sozialen Kommunikation in einem nie gekannten Ausmass her, in dem der Einzelne in ein Netzwerk weltumspannender Kommunikation eingebettet ist. Nur durch die Teilhabe an diesen neuen Formen der sozialen Kommunikation, nur durch die Auseinandersetzung mit anderen Lebensauffassungen, Werthaltung und Weltdeutungen entsteht personale Identität und lässt sich auf Dauer das Profil des eigenen Lebens gewinnen und durchhalten.

Allerdings stellen Pluralität, Unübersichtlichkeit, Widersprüchlichkeit und Konkurrenz der unterschiedlichen Lebensformen, Wertorientierungen und Weltbildern eine besondere ethische Herausforderung für die Erziehung im allgemeinen und für die Medienerziehung im besonderen. Daraus resultieren zunehmende normative Ratlosigkeit und Orientierungsschwäche in der alltäglichen Erziehung, aber auch in der Erziehungswissenschaft. Wie kommen wir zu moralischen Orientierungen in der Medienpädagogik?

\section{Medienethik}

Feste Massstäbe können nicht durch materiale Ethiken vorgegeben werden, z.B. durch Gesinnungs- oder Erfolgsethik (vgl. D.-J. Löwisch, 2000), weil hier der ethischen Permissivität, Gleichgültigkeit, Indifferenz und Relativität fundamentalistisch geantwortet wird. Es bleibt nur das Modell einer diskursiven Verantwortungsethik (vgl. Löwisch 2000, Funiok 2000), also eine formale Ethik. Verantwortung als Prinzip von Ethik muss zu einer Verantwortungshaltung führen, d.h. zu einer inneren moralischen Verfassung des Menschen, als Person verantwortlich handeln zu sollen, nach bestem Wissen und Gewissen.

Eine diskursive Verantwortungsethik für den Bereich der Medien wäre in diesem Sinne als eine Bereichsethik zu konzipieren, die zwei Anforderungen erfüllen müsste. 
- Begründung auf dem Fundament kommunikationswissenschaftlicher Forschung, verbunden mit einer Ausweitung der ethischen Fragenperspektive auf alle Einflussfaktoren, die im Prozess der sozialen Kommunikation zusammenwirken.

- Überwindung eines rein individualethischen Ansatzes; Frage nach den strukturellen Bedingungen, denen der Einzelne im Medienhandeln ausgesetzt ist. Damit ist eine Ausweitung des Begriffs der sozialen Verantwortung verbunden, der auch die aufgaben- und funktionsbezogene Verantwortung sozialer Gruppen, z.B. von Medienunternehmen, berufsbezogener Organisationen oder Medieninstitutionen (Anbieter) umfasst.

Die Analyse von Systembedingungen und Strukturfragen darf aber andererseits nicht dazu führen, dass die innerhalb des Mediensystems tätigen Personen sich von der Verantwortung für das eigene professionelle Handeln weitgehend freigestellt fühlen und auf der anderen Seite dem Rezipienten eine idealisierte Mündigkeit und Autonomie unterstellt wird, der er nicht gewachsen ist, insbesondere als Kind oder Jugendlicher.

\section{Welches sind die grundlegenden Prinzipien einer Medienethik?}

Da es im Bereich der Medien um soziale Kommunikation geht, stehen (nach Schockenhoff 2000) im Zentrum der Bemühungen einer diskursiven Verantwortungsethik die Fragen nach

- der Achtung vor der Wahrheit im Medienhandeln

- der Achtung vor den Kommunikationspartnern.

- Aus dem letzten Prinzip ergibt sich die Notwendigkeit, bei normativen Einzelaussagen der Medienethik alle am Medienprozess beteiligten Akteure im Blick zu haben. Es muss daher ethische Gebote für die Medienproduzenten, für Journalisten, für den Gesetzgeber, für Medienanbieter, Verleger und schliesslich für die Mediennutzer, das Publikum geben.

\section{Medienpädagogische Ethik}

Welche Konsequenzen ergeben sich daraus für eine medienpädagogische Ethik?

Ihre Aufgabe besteht dann darin, bei den Heranwachsenden den Aufbau der Verantwortungshaltung im Umgang mit Medien zu fördern und dafür geeignete Rahmenbedingungen zu schaffen. Das bedeutet letztlich, die Medienpädagogen / Wissenschaftler als Einzelpersonen und die Medieninstitutionen müssen selbst eine solche Verantwortungshaltung gegenüber den Medien und den Heranwachsenden in einer mediengeprägten Alltagswelt ausbilden!

Die zentrale Frage lautet: 
Wie kann und soll das Prinzip einer diskursiven Verantwortungsethik in der Medienpädagogik praktisch werden und in allen medienpädagogischen Berufsfeldern und Tätigkeitsbereichen als Handlungsorientierung dienen?

Medienpädagogik soll bei ihrer Teilhabe an den medialen Kommunikationsprozessen in der Öffentlichkeit und gestützt auf einzelwissenschaftliche Erkenntnisse (Forschungen, Theoriebildung, evaluierte Programme):

- medienpolitisch verantwortlich handeln, d.h. darauf achten, dass der Kinderund Jugendmedienschutz als eine wichtige Möglichkeitsbedingungen für den Aufbau einer Verantwortungshaltung bei Heranwachsenden gesichert ist;

- medienkritisch verantwortlich handeln, d.h., darauf achten ob und dafür Sorge tragen, dass die Medienproduzenten und Anbieter dort, wo sie Kinder- und Jugendliche als Zielgruppen im Blick haben, nicht nur deren Interessen, Bedürfnisse, Wünsche und Fähigkeiten angemessen berücksichtigen, sondern die Inhalte, Angebotsformen und Kommunikationsprozesse als Möglichkeitsbedingungen für den Aufbau einer Verantwortungshaltung bei den Heranwachsenden erkennen und berücksichtigen;

- medienerzieherisch verantwortlich handeln, d.h. den Erziehungspersonen Eltern, Erzieherinnen, LehrerInnen in den verschiedensten pädagogischen Feldern medienpädagogische Kompetenz vermitteln, ihnen Konzepte geben und sie unterstützen in ihrem Bemühen, dass die Heranwachsenden Medienkompetenz und eine Verantwortungshaltung im Umgang mit den unterschiedlichsten Medien aufbauen können.

- medienpädagogisch verantwortlich handeln, d.h. die für eine angemessene Vermittlung von Medienkompetenz bei den Heranwachsenden erforderlichen Kenntnisse gewinnen, sichern und einsetzen, aufgrund welcher Prozesse und unter welchen Bedingungen im Entwicklungsprozess eine mit der Medienkompetenz verbundene Verantwortungshaltung gegenüber den Medien und im Umgang mit den Medien aufgebaut werden kann und welche besondere Rolle dabei die Medien selbst spielen.

\section{Literatur}

Aufenanger, St. (2000): Medienethik und Jugendmedienschutz. In: Ethikbedarf der Medien. ajs-Informationen, 36. Jg. (2000), H. 3, S. 4-9.

Funiok, R.: Medienethik. Der Wertediskurs über Medien ist unverzichtbar. In: «Aus Politik und Zeitgeschichte» (Beilage zu «Das Parlament«). Bonn 6.10.2000, S. 11-18.

Löwisch, D.-J.: Pädagogische Ethik und die Normalität einer diskursiven Verantwortungsethik. In: Pädagogische Rundschau, 54. Jg. (2000), S. 377-384.

Schockenhoff, E.: Zur Lüge verdammt? Politik, Medizin, Justiz, Wissenschaft und die Ethik der Wahrheit. Freiburg, Basel, Wien 2000 (Wahrheit in der demokratischen Öffentlichkeit und in der medialen Kommunikation, S. 265-352). 


\title{
MedienPädagogik
}

Zeitschrift für Theorie und Praxis der Medienbildung

\section{Problemfelder der aktuellen Massenkommunikation und die Suche nach Kriterien}

\author{
Ben Bachmair
}

\section{Interpretationsweisen und Diskurse}

Die aktuelle Umgestaltung der Massenkommunikation läuft auf eine neue MenschMedienbeziehung hinaus, bei der sich Medien in komplexen Arrangements mischen, in denen sie die Funktion von Providern symbolischer Angebote bekommen. Wichtig für die Aneignung dieser komplexen Arrangements ist in Zeiten kultureller Unklarheiten, in denen kulturelle Erfahrungs- und Bewertungskontexte fehlen, welche Interpretations-Folie die Produktion und welche die Diskurs-Flächen (-zeiten) die Sender anbieten. Nicht was angeboten wird, ist die vorrangige kulturelle und pädagogische Frage, sondern das Wie! Und diese Frage sollte nicht im Sinne der Individualisierung kultureller Risiken an die Mediennutzern delegiert werden.

Beispiel ist hierzu bei Big Brother die Unklarheit, welche Zeichenfunktion die Akteure im Container haben, z.B. als ge-castete Typen. Was ist Zeichen, was ist Thema? Die Semiotik hat dafür das hübsche Schlagwort von der Krise der Repräsentation formuliert.

\section{Herrschaft und Legitimation}

Die theoretisch rührend hilflose und praktisch folgenlose Frage nach der Herrschaftsausübung im Zusammenhang mit einem neuen Genre wie Big Brother oder der Ruf nach Jugendschutz, als Talkshows mit der Funktionalisierung von Alltagsakteuren aus dem Ruder liefen, ist ein Indiz, wie wenig die politische Frage in Sachen Massenkommunikation trotz demokratischer Grundordnung auf der öffentlichen Agenda steht. Gäbe es nicht Wirtschaftsstaatsanwälte, die beispielsweise EM-TV unter die Lupe nehmen, reduzierte sich öffentliche Kontrolle und Legitimation der Akteure im Mediengeschäft auf die Aufsichtsräte der GmbHs.

Die Verletzlichkeit der Menschen (Anthony Giddens) und die empathische Parteinahme für die Unerfahrenen

Die Idee der Humanität mit ihren Formen der Fürsorge, Anteilnahme und Solidarität ist in einer kulturell und hier wiederum «medial» sich definierenden Gesellschaft mit zwar standardisierten dennoch unklaren Repräsentionsformen neu zu deuten. Die Basis ist eine Alltagshermeneutik, bei der die Menschen in der Spannung von Ichstärke, Alltagsgestaltung und Wirklichkeitsbezug sowohl auf Empathie vertrauen als auch Empathie aufgrund ihrer entwickelten Interpretationsweise einbringen können. 
Wie sind die Fragen nach Interpretationsweisen und Diskurse, Herrschaft und Legitimation, Verletzlichkeit und Empathie in eine pädagogischer Sichtweise zu übersetzen? Das reicht vom Eigensinn der Kinder und Sachen bis zum Anker in einer fliessenden Welt der Zeichen.

Die neue und eher konsumierende, mediengerechte Form der Aneignung hat eine postmoderne Dynamik, die nichts mehr mit Pauken zu tun hat. Kinder eignen sich die Welt in einer spezifischen Erlebnisweise an. Diese Erlebnisweise hat sich mit Konsumorientierung und Individualisierung entwickelt, bei der Medien, insbesondere Fernsehen, einen nicht zu unterschätzenden Anteil beitrugen. Die heutige Kindergeneration ist im Trend zur Individualisierung und mit der Notwendigkeit aufgewachsen, individuelle Lebenswelten gerade auch mit den symbolischen Materialien der Medien aufzubauen. Damit beginnen Kinder und Jugendlichen ihre Erlebnisweisen zu verändern. So stehen sie tendenziell im Mittelpunkt einer eigenen, persönlichen Welt, die sich anders aufbaut als die Lern- und Sachwelt der Schule.

Diese postmoderne Welt entsteht um das eigene Ego in Prozessen der Bedeutungskonstitution. Bedeutungskonstitution ist das Gegenmodell zur passiven Medienrezeption. Kinder eignen sich das symbolische Material von Konsumobjekten, Medien und Ereignissen individuell an, indem sie ihnen perspektivisch und innerhalb von Relevanzrahmen subjektive Bedeutung verleihen. Die jeweilige Medienwelt ist einer dieser Relevanzrahmen.

\section{Sich vergewissern: Reflexivität für Bastler in Kulturinszenierungen}

Medienkompetenz ist eine Form der reflexiven Beziehung von Kinder zu Medien. Darüber hinaus gibt es vermutlich eine Fülle von Möglichkeiten, wie Medien und Schule Kindern emotionale, soziale und rationale Distanz und Auseinandersetzung anbieten. Auf einer weiteren Stufe von Reflexivität sind Angebote wichtig, die dazu beitragen, Lebenswelten selber zu ordnen und zu gestalten. In diesem Kontext sind beispielsweise die Hörgeschichten «Zwischen Herrn Morens Ohren» interessant, weil sie mit Rhythmus nicht nur einen Beitrag zur Ordnung einer komplexen Welt anbieten, sondern zeigen, wie der hörbare Rhythmus der Ohren der selbstverständlichen Dominanz der Augen gewachsen ist. Reflexivität ermöglichen aber auch Zwecktexte wie die der Werbung, wenn sie die simple Funktion von Werbung als Kaufanreiz oder Produktbotschaft durchbrechen und die Werbeelemente als Spiel- und Ästhetikmaterial anbietet. 


\section{Eigensinn von Kindern, Dingen und Ereignissen}

In ihrem Verhältnis zu Medien sind Kinder sehr wohl kreative Schöpfer, wobei Schöpfung ein Prozess der Bedeutungskonstitution ist. Sie schaffen Bedeutung in ihrer eigenen Sinnperspektive. Die Kinder-Medien-Beziehung ist selbstverständlich auch eine medienpolitisch und ökonomisch bestimmte Beziehung, in denen Kinder nur vom Marketing auskalkulierte Abnehmer sind, die letztlich nur als Quoten fungieren. Kinder sollen eben nicht zu Objekten von Zwecktexten oder Einschaltquoten werden. Ihr Eigensinn in der Doppelbedeutung von Widerständig und Sinnschaffen ist deshalb eine wichtige Bewertungsdimension für Fernsehangebote. Daraus ergibt sich die Leitfrage, ob und wie Schule, Sender Warenproduzenten, Entertainment-Unternehmen usw. Kinder als Subjekte adressieren, welches implizite Kinder- und Konsumentenbild sie unterstellen. Gleichzeitig existiert auch in der erlebnisorientierten Gesellschaft die Welt der Sachverhalte und Ereignisse als etwas Eigenständiges mit eigenem Wert und Sinn, das über das individuelle Verfügen hinaus reicht. Es geht in dieser Bewertungsdimension also um die Funktion von Medien für die Realitätserklärung, deren konsequente Form das Lehren und Lernen ist.

\section{Kontexte herstellen in der Welt als Supermarkt}

Die Aufgabe, sich in einer vielschichtigen und auch brüchigen Welt die eigene Lebenswelt herzustellen, ist nicht nur für Kinder eine kräftezehrende und riskante Aufgabe, zu der sie Hilfe brauchen. Nicht erst mit der Globalisierung liefert Fernsehen bzw. andere elektronische Medien eine Fülle von Formaten und Genres, die sich nicht allein auf der Basis kultureller Tradition erklären. Gängig ist in diesem Zusammenhang die Vorstellung, Kinder sollten beim Fernsehen natürlich nicht allein gelassen werden; zuhörenden, erklärende oder auch beruhigenden Eltern bzw. Bezugspersonen sollten sie dabei begleiten. Dies nicht allein der Familie zu überlassen, ist auch Aufgabe der Medienanbieter. Es gilt insbesondere, wenn Programme den angestammten Kulturkontext verlassen und Kinder sie ohne tradierten oder vertrauten Bezugsrahmen, also ohne eigene Genrekompetenz, rezipieren.

Dazu gehört sicherlich etwas, das Kinderfernsehen, z.B. die «Sendung mit der Maus«, immer schon macht, die Welt zu erklären. Neu sind zwei andere Wege, nämlich über Bezüge zu Lebensstilen und mit typischer Alltagsästhetik eine symbolische Brücke zu neuen sozialen Gefüge wie Fan-Gruppen, Szenen, Milieus zu bauen. So bietet der Bezug zu Stilen und Milieus soziale Verortung. Warum sollte nicht auch die Schule, Kindern und Jugendlichen Ordnung und stilistische Orientierung anbieten? 


\section{MedienPädagogik}

Zeitschrift für Theorie und Praxis der Medienbildung

\section{Instantwissen und Bricolage \\ - Überlegungen zu einigen ethischen Implikationen}

Theo Hug

\section{Ausgangslage}

Die Medienpädagogik ist seit einigen Jahren mit erheblich veränderten Problemlagen und Sozialisationsbedingungen konfrontiert. So entstehen gegenwärtig im Zuge differenzierter Globalisierungsprozesse neue Kommunikationskulturen und Formen des «In-der-Welt-seins» (vgl. Volkmer 1998). Entsprechende Fragestellungen einer «Medienpädagogik in der Globalisierung» (vgl. Hug 2000) wurden bislang erst ansatzweise bearbeitet. Die hier anvisierten Entwicklungsdynamiken betreffen die Frage des Wissens.

Hand in Hand mit den beschleunigten Lebensverhältnisse sind Formen des 'Wissens auf den ersten Blick' entstanden, die zunächst an schulisches Abfragewissen erinnern mögen (vgl. Rumpf 1971). Das in den sogenannten «Millionen»-Shows gefragte Wissen mag als aktuelles und prototypisches Beispiel gelten. Als kleinster gemeinsamer Nenner der verschiedenen Varianten von Instantwissen lassen sich die Merkmale vielseitige Brauchbarkeit, leichte Verständlichkeit, Unterhaltsamkeit und umfassender Erklärungsanspruch benennen, wobei u.a. gemeinsam geteiltes Massenwissen, situationsbezogenes Sofort-Wissen, unproblematisches Gebrauchswissen zur Erlebnisintensivierung oder etwa fragmentiertes Weltwissen unterschieden und näher charakterisiert werden können. Insgesamt haben diese Wissensformen wie auch die Bricolage-Varianten (Bsp. fallbezogenes Reflexionswissen oder Handlungswissen für den improvisierenden Umgang mit begrenzten Ressourcen) in Relation zu den Formen "gesicherten» Langzeit- oder Planungswissens an Bedeutung gewonnen (vgl. Hug / Perger 2000).

\section{Ethische Überlegungen}

Auf dem Hintergrund dieser Ausgangslage lassen sich ethische Implikationen formulieren, die hier thesenartig skizziert werden:

\section{Wissens(kluft)-Gesellschaft oder urteilsfähige Mediengesellschaft}

Die Zugriffsmöglichkeiten auf Datenbestände und Medienangebote haben sich enorm vergrössert, ohne dass sich damit die individuellen Wissensstände erhöht hätten. Die Rede von neuen virtuellen Welten, Wissensdimensionen und Erfahrungsqualitäten erscheint eher als Teil einer kollektiven Fiktion oder werbewirksamer Gag 
denn als erfahrbare Wirklichkeit. Die Entwicklung einer wissens- und medienspezifischen Urteilskompetenz erweist sich hier als unumgänglich, wenn das Diktum einer zweigeteilten Gesellschaft Programmierern und Programmierten an Bedeutsamkeit verlieren soll.

\section{Kommerzialisierung und Sozialpflichtigkeit des Wissens}

die Entwicklung einer Urteilsfähigkeit in Bezug auf Informationsqualitäten und Wissensformen muss auf möglichst breiter Basis möglich sein. Die neuen Informationstechnologien erzeugen nicht «von selbst» eine humanere Kommunikationskultur. Da sich die Ökonomie kaum einer Ethik der Sozialpflichtigkeit des Wissens unterwerfen wird, sind hier tendenziell pragmatische Lösungen (statt fundamentalethische Begründungen) anzustreben, die de facto einen Kompetenzerwerb beispielsweise zur Unterscheidung problematischer instantaner Formen ermöglichen.

\section{Medialität des Wissens und Medienkompetenz}

An die Stelle des Traums von der vormedialen Unschuld kann die Orientierung an der Unterscheidung von symbolischen Formen und Ordnungen treten. Medialität ist keine optionelle oder periphere, sondern eine zentrale Bestimmung des menschlichen Wahrnehmens, Denkens, Handelns und Wissens. So können insbesondere Realitätsbehauptungen reflektiert und differenziert betrachtet werden. Auf dem Weg von der «wahren Wirklichkeit» zur «Modalisierung der Wirklichkeitserfahrung» (vgl. Schmidt 1994, S. 277 ff) gilt es eine mehrperspektivische Kompetenzentwicklung vor allem im Hinblick auf zwei Gesichtspunkte voranzubringen: Einmal geht es um eine integrative Entwicklung der verschiedenen sozial-kommunikativen, pragmatischtechnischen, reflexiv-theoretischen und selbstreflexiven Kompetenzdimensionen in Relation zu Lebensabschnitten, sozio-kulturellen Problemlagen und spezifischen Anwendungskontexten, und zum Zweiten um die Befähigung zum Übergang zwischen Medienwelten und Wirklichkeitsbereichen.

Die Medienpädagogik, in deren Zuständigkeit die Bearbeitung die Schnittstellen des Aufwachsens, der Arbeit, der Bildung, der Erziehung, des Lernens und der Verständigungsprozesse zu den medialen Entwicklungen fällt (vgl. Hug 2001), ist dabei in einem basalen Dilemma: Einerseits ist sie im Unterschied zu anderen Bereichen der Medientheorie, -praxis und -produktion immer wieder aufgefordert ethische und normative Dimensionen zu bearbeiten, andererseits ist die Problematik der relativen Konsequenzenlosigkeit ihrer Bemühungen oder deren Merkmale des «Zuspät-Kommens» oder der ungewollten Anpassungsleistung unübersehbar. Trotzdem muss der Verzicht auf die Romantisierung 'vormedialer' Zeiten nicht auf einen 
Kulturpessimusmus hinauslaufen. Wenn wir akzeptieren, dass gelingendes Aufwachsen nicht jenseits, sondern nur innerhalb der Medienwelten stattfinden kann, dann eröffnen sich mit den medialen Wahrnehmungsdisziplinierungen und Beschränkungen menschlicher Ausdrucksmöglichkeiten, immer auch Handlungs- und Gestaltungsspielräume, die es zu kultivieren gilt (vgl. Wiegerling 1998, S. 235). Das Kriterium des Gelingens scheint am ehesten dann einlösbar zu sein, wenn konkrete Normen und Werte Gegenstand diskursiver Auseinandersetzungen bleiben und jeweils auf Zeit ausgehandelt werden. Der Versuch einer dauerhaften Festschreibung derselben dürfte jedenfalls eher einer Art kollektiven Gewissensberuhigung weisser WohlstandspädagogInnen (vgl. Herra 1988) als der Weiterentwicklung der Medienkompetenz möglichst breiter Bevölkerungsgruppen dienlich sein.

\section{Literatur}

Herra, R. A.(1988): Kritik der Globalphilosophie. In: Wimmer, F. M. (Hrsg.): Vier Fragen zur Philosophie in Afrika, Asien und Lateinamerika. Wien, S. 13-33.

Hug, Th. (Hrsg.) (2000): Medienpädagogik in der Globalisierung. Themenschwerpunkt der Zeitschrift SPIEL, Nr. 9 / H. 2, 2000.

Hug, Th. (2001): Medienpädagogik - Begriffe, Konzeptionen, Perspektiven. In: Rusch, G. (Hrsg.): Einführung in die Medienwissenschaft. Opladen (im Erscheinen).

Hug, Th./ Perger, J. (2000): Instantwissen und Bricolage. Wissensformen in der westlichen Medienkultur. Hagen: Studienbrief der FernUniversität.

Rumpf, H. (Hrsg.) (1971): Schulwissen. Probleme der Analyse von Unterrichtsinhalten. Göttingen.

Schmidt, S. J. (1994): Kognitive Autonomie und soziale Orientierung. Konstruktivistische Bemerkungen zum Zusammenhang von Kognition, Kommunikation, Medien und Kultur. Frankfurt/M.

Volkmer, I. (1998): «Hic et nunc» von Nachrichtengenerationen. Überlegungen zu der Kategorie des «Da-seins» aus der Sicht globaler Phänomenologie. In: Hug, Theo (Hrsg.): Technologiekritik und Medienpädagogik. Zur Theorie und Praxis kritisch-reflexiver Medienkommunikation. Baltmannsweiler, S. 167-179.

Wiegerling, K. (1998): Medienethik. Stuttgart/ Weimar. 


\title{
Ethische Implikationen einer handlungs- und entwicklungsorientierten Medienpädagogik
}

\author{
Bardo Herzig und Gerhard Tulodziecki
}

Ethische Implikationen einer handlungs- und entwicklungsorientierten Medienpädagogik ergeben sich zunächst aus zwei - thesenartig formulierten - Überlegungen:

- Die Nutzung vorhandener Medienangebote und die eigene Gestaltung von Medienbeiträgen stellen Formen menschlichen bzw. sozialen bzw. kommunikativen Handelns dar. Sie unterliegen damit dem anthropologischen Grundverhältnis des Menschen im Sinne des Verhältnissen zu sich selbst, zur dinglichen und zur sozialen Umwelt.

- Handeln im Medienbereich lässt sich - wie Handeln generell - durch folgende Modellvorstellung charakterisieren (vgl. Tulodziecki 1997, S. 116 ff.): Durch die Wechselbeziehung zwischen situativen Anforderungen und grundlegenden Bedürfnissen kommt es zu einem Spannungszustand, der durch die Wahl einer Handlungsmöglichkeit im Sinne eines angestrebten Zustandes aufgelöst werden kann. Welche und wie viele Handlungsmöglichkeiten ins Bewusstsein kommen und in welcher Weise sie bedacht werden, hängt vom Erfahrungs- und Kenntnisstand des Individuums sowie von seinen intellektuellen Fähigkeiten ab. Welche Bewertungen vorgenommen werden, wird durch sozial-moralische bzw. ethische Orientierungen beeinflusst. In der Wechselwirkung von Situation, Bedürfnissen, angestrebtem Zustand, Erfahrungs- und Kenntnisstand sowie intellektuellen Fähigkeiten und sozial-moralischen Orientierungen wird eine Handlungsmöglichkeit ausgeführt. Diese hat bestimmte Folgen, die verarbeitet werden und auf die genannten Faktoren des Handlungsmodells zurückwirken.

Bezogen auf dieses Handlungsmodell wird die Frage bedeutsam, welche sozial-moralischen bzw. ethischen Orientierungen beim Medienhandeln eine Rolle spielen und wie sie entwickelt werden können. Vor diesem Hintergrund lassen sich vier weitere Thesen formulieren:

- Sozial-moralische bzw. ethische Orientierungen können danach charakterisiert werden, inwieweit über die eigene Sichtweise hinaus die Perspektive anderer eingenommen und inwieweit Verantwortung für das Zusammenleben mit anderen übernommen wird sowie welcher Begriff von richtigem bzw. gerechtem Handeln zugrunde liegt (vgl. Herzig 2001b; Tulodziecki 1997, S. 134 ff.)

- Auf der Basis solcher Charakterisierungen kann man in Anlehnung an Mead und Kohlberg unterschiedliche Stufen sozial-moralischer bzw. ethischer Entwicklung beschreiben: Egozentrische Fixierung auf die eigenen Bedürfnisse unter Vermeidung von Strafe, Orientierung an den eigenen Bedürfnissen unter Beachtung der 
Bedürfnisse anderer, Orientierung an der Erwartung von Bezugspersonen und Bezugsgruppen, Orientierung am sozialen System mit einer bewussten Übernahme gerechtfertigter Verpflichtungen, Orientierung an individuellen Rechten und ihrer kritischen Prüfung unter dem Anspruch der menschlichen Gemeinschaft. Damit ist insbesondere das Verhältnis des Individuums zur sozialen Umwelt angesprochen.

- In Ergänzung zur gerechtigkeitsorientierten Ausrichtung moralischer Urteilsfähigkeit bei Kohlberg lassen sich im Anschluss an Gilligan Phasen moralischer Orientierung formulieren, die Verantwortung und Empathie in den Mittelpunkt stellen. Gilligan berücksichtigt dabei ausdrücklich das Verhältnis des Menschen zu sich selbst. Beide Modelle lassen sich in einem integrativen Ansatz wechselseitig in ergänzender Weise aufeinander beziehen (vgl. Herzig 1998, S. 107 ff., 139 ff.).

- Das Verhältnis des Menschen zur Sachwelt als dritter Teil des anthropologischen Grundverhältnisses kann - im Hinblick auf die ethische Dimension - als Bedingungsfaktor ebenso wie als Konsequenz interpretiert werden.

- Solche Orientierungen werden in Situationen der Nutzung vorhandener Medienangebote und bei der eigenen Gestaltung von Medienbeiträgen sowie bei der Wahrnehmung und Bewertung von Medieninhalten wirksam. Unter der Zielvorstellung eines sozial verantwortlichen Handelns im Medienbereich geht es darum, im Zusammenhang der Auseinandersetzung mit Medienfragen stets auch die Entwicklung der sozial-moralischen bzw. ethischen Orientierungsfähigkeit zu fördern.

- Der Entwicklungsprozess soll dabei von einer egozentrischen Perspektive bis zu einer menschheitsbezogenen Perspektive, von der Verantwortung für das eigene Wohl bis zur Verantwortung für das Wohl aller und von einer Orientierung an Bestrafung und Gehorsam bis zu einer Orientierung an universalen ethischen Prinzipien reichen.

- Entwicklungsförderung in sozial-moralischer bzw. ethischer Hinsicht setzt dabei eine angemessene Unterscheidungsfähigkeit zwischen unterschiedlichen sozialen Perspektiven, zwischen unterschiedlichen Verantwortungsfeldern und zwischen gerechtfertigtem und ungerechtfertigtem Handeln - d.h. eine angemessene intellektuelle Entwicklung - voraus. Sie kann durch die Auseinandersetzung mit sozialen Konflikten bzw. Wertkonflikten im Medienbereich gefördert werden. Für die Überbrückung der Differenz zwischen kognitivem oder auch emotionalem Urteil und tatsächlicher Handlungsbereitschaft empfiehlt sich die kommunikative Exploration von Lösungsszenarien (vgl. Herzig 1998, S. 329 f.; Herzig 2001a). 


\section{Literatur}

Herzig, B. (1998): Förderung ethischer Urteils- und Orientierungsfähigkeit. Grundlagen und schulische Anwendungen. Münster, New York.

Herzig, B. (2001a): Medienhandeln und Verantwortung. Zur Förderung eines verantwortungsbewussten Umgangs mit (neuen) Medien. In: Schweer, M. (Hrsg.): Der Einfluss der Medien. Vertrauen und soziale Verantwortung. Opladen, S. 197-220.

Herzig, B. (2001b): Werterziehung in der Schule. Eine erwägungsorientierte Auseinandersetzung mit Lawrence Kohlberg. In: Loh, W. (Hrsg.): Erwägungsorientierung in Philosophie und Sozialwissenschaften. Stuttgart, S. 79-108.

Tulodziecki, G. (1997): Medien in Erziehung und Bildung. Grundlagen und Beispiele einer handlungs- und entwicklungsorientierten Medienpädagogik. 3. Aufl., Bad Heilbrunn. 


\title{
Aspekte der Medienethik in der Lehrerprofessionalisierung
}

\author{
Manfred Lang
}

Professionelles Lehren und Lernen erfordert Autonomie, die dem Lehrerberuf in den vergangen Jahrhunderten weder unter kirchlicher noch staatlicher Aufsicht zugestanden wurde. Insbesondere der Autonomie für eine professionelle Ethik standen Mechanismen der Kontrolle durch Moralisierung, Standardisierung oder Einrichtung ethischer Codes für Lehrerhandeln gegenüber (Terhart, 1998). Es stellt sich daher die generelle Frage, wie professionelle Ethik im Informationszeitalter in einem Prozess der Selbstentwicklung in Netzwerken und selbstgesteuerten interaktiven Systemen möglich ist.

Terhart sieht Professionalisierung als Enkulturation des Selbst: Die moralische Dimension entwickelt sich gleichzeitig neben einer kognitiven und praktischen Dimension der Professionalität:

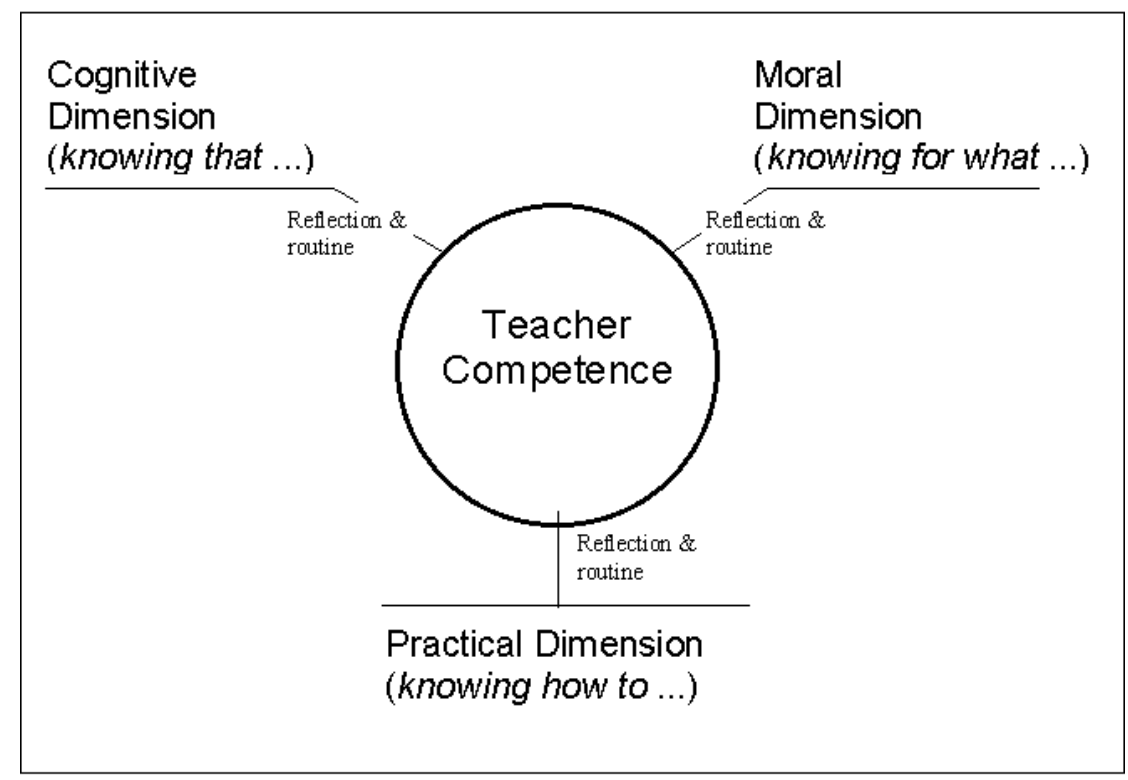

Abb. 1.: Modell professioneller Entwicklung von Lehrkräften nach Terhart (1998)

Unterricht vermittelt und reflektiert Wissen und Fertigkeiten für einen angemessenen ethischen Zweck. Unterricht als moralische Aktivität ist jederzeit mit professionellen Handlungen oder Strukturen eines «hidden curriculum» verbunden, auch wenn Lehrende und Lernende den moralischen Einfluss nicht immer wahrnehmen. Moralische Handlungen äussern sich in Reglementierungen, Bewertungen und Einübung von Tugenden aber auch in Entscheidungen für bestimmte fachliche Inhalte als Vehikel 
moralischen Einflusses auf Schülerinnen und Schüler (Sockett, 1992). Beispiele hierfür sind Aufforderungen wie «Nicht vom Nachbarn abschreiben» oder «Sich melden», der Stil der Lehreräusserungen zum Schülerverhalten oder die Auswahl von naturwissenschaftlichen oder literarischen Inhalten nach C. P. Snows zwei Kulturen. Medienpädagogische Ethik ist entsprechend eingebunden in eine Unterrichtspraxis, die nicht als gesonderte Dimension in Erscheinung treten muss. Sie ist ein integraler Teil eines medienunterstützten Vermittlungsprozesses, durch den Kenntnisse und Fertigkeiten über Medieninteraktionen eine besondere Bewertung erfahren. Das Watzlawicksche Axiom bekommt damit auch die spezifische Bedeutung «Man kann nicht nicht medienethisch kommunizieren».

Doch wie kann professionelles ethisches Handeln als Enkulturation entwickelt werden? Die festgestellte Voraussetzung der Autonomie für professionelles Handeln muss auch für den Teilaspekt der moralischen Dimension gelten. Hierzu ist nach Terhart eine frühzeitige Auseinandersetzung der Betroffenen mit Grenzen des professionellen Engagements und ethischer Probleme zur Sensibilisierung schon während der ersten Jahre der Lehrerausbildung notwendig.

Neben der Enkulturation ist zudem für interaktive und vernetzte Medien eine Professionalität durch Dialog in einer Lerngemeinschaft von besonderer Bedeutung (Colnerud et al. 1999). Das beinhaltet eine diskursive Lernkultur ohne Machtausübung in der Argumentation zwischen Lehrenden und Lernenden und Kollegialität und Partnerschaft auch im weiteren schulischen Bereich. Hier werden Modelle ethischen Handelns angeführt, die auf Kohlbergs (1985) «Just Community» aufbauend für eine teilnehmende Demokratie und lokale Selbstbestimmung und gegen Machtausübung und Indoktrination stehen. Professioneller Umgang mit interaktiven und vernetzten Medien setzt entsprechend eine Ethik der Kooperation, Verantwortung und partnerschaftlichen Achtung in Lerngemeinschaften voraus. Er ermöglicht eine gemeinschaftliche Wissensbildung im Gegensatz zur reproduktiven Aneignung des Einzelnen. Neue Entwicklungen der Informations- und Kommunikationstechnologien können Lehrende und Lernende in Netzwerken kooperativer Lerngemeinschaften unterstützen (Computer Supported Collaborative Learning Networks, Simons et al. 2000) und zu interaktiven Formen der ethisch reflektierten Wissensbildung in der Schule und zwischen verschiedenen Lerngemeinschaften führen. Medienethik ist in diesem Sinne nicht Gegenstand einer formalen Vermittlung, sondern Teil eines diskursiven Prozesses mit ethischen Voraussetzungen für eine Lerngemeinschaft. Ein Modell professionellen Umgangs mit Medien erfordert damit eine Erweiterung des Modells von Terhart um eine kooperative Dimension, in der ethische Fragen ein konstituierendes Element sind. Medienethik in der Lehrerbildung setzt entsprechend eine besondere Struktur kooperierender Lerngemeinschaften voraus, in der durch Dialog und Reflexion ethisches Handeln bewusst gemacht und für Unterrichtspraxis vorbereitet wird. 


\section{Literatur}

Colnerud, G./ Clark, C./ Klette, K./ Kristiansen A. (1999): Moral Life in Schools and the Challenges to Teacher Professionalism. In: Lang, M./ Olson, J./ Hansen, H./ Bünder W. (Eds.): Changing schools/ changing practices: Perspectives on educational reform and teacher professionalism. Louvain, pp. 99-107.

Kohlberg, L. (1985): The Just Community Approach to Moral Education. In: Berkowitz, W./ Oser, F. (Hrsg.): Moral Education: Theory and Application. London, pp. 27-87.

Simons et al. (2000): Computer Supported Collaborative Learning Networks in Primary and Secondary Education. Final Report. http://improving-ser.sti.jrc.it/default/.

Sockett, H. (1992): The Moral Aspects of the Curriculum. In: Jackson, P. (Ed.): Handbook of Research on Curriculum. New York, pp. 543-568.

Terhart, E. (1998): Formalized Codes of Ethics for Teachers: between professional autonomy and administrative control. European Journal of Education, 33(4), 433-443. 


\section{MedienPädagogik}

Zeitschrift für Theorie und Praxis der Medienbildung

\section{Ethische Aspekte heteronomer und autonomer Moral internetbasierter Kommunikation}

Rudolf Kammerl

Die ethische Reflexion der expliziten und impliziten Handlungsnormen im Bereich der medienvermittelten Kommunikation und Information beinhaltet auch die Aufgabe, zu untersuchen, inwiefern Strukturen von Kommunikation moralisches Handeln begünstigen oder erschweren. Im Anschluss an internetethische Fragestellungen werden im Folgenden Formen heteronomer und autonomer Moral internetbasierter Kommunikation einer genaueren Betrachtung zugeführt.

\section{Anonymität im virtuellen Raum begünstigt unmoralisches Verhalten}

Im Bereich der medialen Freizeitgestaltung erfreut sich die internetbasierten Kommunikation mittels Chat, Mail und News grosser Beliebtheit. Der Austausch über unterschiedlichste Themen mit völlig unbekannten Internetnutzern, sowie das gegenseitige Kennenlernen stellt für viele einen besonderen Reiz dar. Internetnutzer berichten jedoch auch über eine Vielzahl von Verstössen gegen einfache moralische Prinzipien zwischenmenschlicher Kommunikation Diese reichen von kleineren oder grösseren Unhöflichkeiten - die eher als Frage der Etikette abgetan werden könnten - über Lügen, Beleidigungen, gezielten Irreführungen bis hin zu harten verbalen Attacken (z. B. im Sinne von sexuellem Missbrauch) und informatischen Attacken (z.B. flooding, mailbombs etc.). Diese Verhaltensweisen werden vor allem durch die Möglichkeiten, als Internetnutzer seine Anonymität zu wahren, und - damit einhergehend - durch das weitgehende Fehlen von nachhaltigen Sanktionsmöglichkeiten begünstigt.

\section{Heteronome und autonome Moral in der netzbasierten Kommunikation}

Für immer mehr Jugendliche wird die Kommunikation im Internet Bestandteil der medialen Sozialisation. In ihrer moralische Entwicklung befinden sie sich im Übergang von heteronomen zu autonomen Moralvorstellungen. Ausgehend von den Untersuchungen zur moralischen Entwicklung im Anschluss an Piaget und Kohlberg gilt es zu untersuchen, welche Formen heteronomer und autonomer Moral und welche der jeweils hierfür günstigen Strukturbedingungen (wie z. B. Chancen zu Teilnahme an kooperativen Entscheidungsprozessen, stabile emotionale Zuwendung, soziale Anerkennung vs. Geringschätzung, Indifferenz oder Unberechenbarkeit in der Wertschätzung, eingeschränkte Kommunikationsmöglichkeiten, überzogene Restriktion, sowie unklare oder inadäquate Verantwortungszuschreibungen) in den Konstellationen internetbasierter Kommunikation vorzufinden sind. 
Das Internet mit seinen unterschiedlichen Diensten ist hierbei differenziert zu betrachten. Chatten (plaudern, schwätzen) ist eine synchrone Kommunikationsform. Die Gesprächspartner schreiben sich mehr oder weniger zeitgleich Texte, die in nahezu Echtzeit auf dem Bildschirm des Gegenüber zu sehen sind. Der Schreibstil ähnelt dem der mündlichen Konversation sehr. Soweit bei dieser Form netzbasierter Kommunikation Konversationsregeln explizit ausgewiesen werden (z.B. in FAQs, Netiquette, Chatiquette), sind sie meist fest vorgegeben und kaum verhandelbar. Ihre Einhaltung wird nicht selten überzogen restriktiv von einzelnen Gruppenmitgliedern und/ oder dem Moderator überwacht. Normverstösse können u.a. zum Ausschluss aus dem Kommunikationskontext führen. Die Kommunikationsmöglichkeiten sind sehr eingeschränkt, Meta-Kommunikation findet kaum statt und wird häufig von den Teilnehmern als lästig empfunden. Anonymität, Distanz und die eingeschränkte nonverbale Kommunikation lassen die Internetnutzer lange über die tatsächliche gegenseitige Wertschätzung im Unklaren. Freizeitchats weisen tendenziell eine hohe Häufigkeit von moralisch problematischen Verhalten auf. Im Hinblick auf die Moralentwicklung von Jugendlichen muss der - insbesondere unmoderierte - Chatroom tendenziell als problematischer Raum medialer Sozialisation beschrieben werden.

Natürlich muss auch auf die andere Seite hingewiesen werden: Im Chat können sich auch ganz gelungene Sozialbeziehungen entwickeln. Starke subjektive Relevanz der Kommunikation mit anderen Internetnutzern ist Vorraussetzung dafür, dass sich stabile Beziehungen herausbilden können, in denen Wertschätzung und Anerkennung erfahrbar wird. Virtuelle Kontakte können soziale Gratifikationen bieten und neue Gestaltungsräume eröffnen. Empathie und Bindung im Einzel- oder Gruppenkontakt führen zu prosozialen Orientierungen aus denen auch soziale Beziehung ausserhalb des Netzes entstehen können.

\section{Virtuelle Identität - virtuelle Moral?}

Die Informationen über die Identität der Internetnutzer sind sehr reduziert, können weitgehend vom Kommunikator modifiziert werden und werden vom Adressaten häufig verzerrt wahrgenommen. Für die Frage nach der individuellen Orientierung an etwaigen moralischen Maximen ist die Bedeutung des Handelns im Internet für das Selbstkonzept entscheidend. Für das Selbstkonzept der Internetnutzer kann netzbasierte Kommunikation sowohl funktional im Sinne experimenteller Identitätsarbeit sein, aber auch Realitätsflucht, Spiel oder authentische Selbstdarstellung sein. Die Phänomen «virtuelle Identität» kann - im Rahmen postmoderner Identitätskonzepte (Multiple Identität, Patchworkidentität) - als mehr oder weniger entkoppelte Teilidentität interpretiert werden oder - im Rahmen moderner Identitätskonzepte - als Kommunikationsfeld einer Gesamtidentität. Bei geringer Identitätsrelevanz netzbasierter Kommunikation ist mit Abbruch der Kommunikation, mit Wechseln der 
Virtuellen Identität und höherer Bereitschaft zu Normverstössen zu rechnen. Eine Orientierung an bereichsübergreifenden individuellen moralischen Überzeugungen ist insbesondere dann zu erwarten, wenn es sich bei der internetbasierten Kommunikation um eine authentische und stabile handelt, mit hoher Identitätsrelevanz für alle Beteiligten. Bindung zwischen den Internetnutzern und die Wahrnehmung des Internet als moralisch bedeutsames Handlungsfeld sind hier ebenfalls als begünstigende Faktoren zu nennen.

\section{Wie können medienethische Massstäbe für die internetbasierte Freizeitkommuni- kation Geltung erlangen?}

Medienethik betreibt ethische Reflexion der Handlungsnormen im Bereich der medienvermittelten Kommunikation und Information und versucht moralische Urteile und geforderte Verhaltensweisen rational zu begründen und auf verallgemeinerbare moralische Prinzipien zurückzuführen. Medienethik geht also über eine neutrale Beschreibung vorfindbarer Moral und Handlungsregeln hinaus und formuliert eigene normative Bewertungsmassstäbe für den Medienalltag.

Bei der Freizeitgestaltung internetbasierte Kommunikation mit zunächst unbekannten Netzteilnehmern überwiegen emotionale Orientierungen. Spielerische Aspekte, irrationale Zuschreibungen und Stereotypisierung, sowie daraus resultierende emotionale Überreaktionen (Flames) prägen die Verlauf virtueller Kontakte und die Entwicklung daraus entstehende Beziehungen sehr. Statt rationale begründbarer moralischer Prinzipien bestimmen emotionale Befindlichkeiten die Moral des individuellen Handelns.

Die Kommunikationskultur des Internet kann nicht als eine einheitliche beschrieben werden. In dem weltweiten Medium Internet sind sehr heterogene Subkulturen mit kontextspezifischen Konventionen aufzufinden. Es ist anzunehmen, dass sich medienethische Maximen bei den Internetnutzern als Bestandteil handlungsanleitender Individualethiken nur dann etablieren können, soweit es innerhalb und ausserhalb des Mediums gelingt, mittels diskursiver Verfahren hierfür Verbindlichkeit einzufordern. Es ist zu erwarten, dass dies nur im Kontext einer allgemeinen moralischen Bildung gelingen kann. 